\title{
Edad y crecimiento del dorado Coryphaena hippurus, en el Golfo de Tehuantepec, México
}

\author{
Age and growth of dolphinfish Coryphaena hippurus, \\ in the Gulf of Tehuantepec, Mexico
}

\author{
Carmen Alejo-Plata ${ }^{1}$, José L. Gómez-Márquez² e Isaías H. Salgado-Ugarte²
}

\begin{abstract}
${ }^{1}$ Universidad del Mar, Ciudad Universitaria, Puerto Ángel, San Pedro Pochutla, CP 70902, Oaxaca, México. plata@angel.umar.mx ${ }^{2}$ Facultad de Estudios Superiores Zaragoza, Universidad Nacional Autónoma de México, Av. 5 de Mayo y Fuerte de Loreto, Col. Ejército de Oriente, Iztapalapa, Apartado Postal 09230 CP 9-020, México D.F., México
\end{abstract}

\begin{abstract}
The dolphinfish Coryphaena hippurus, is one of the main species caught by the artisanal fisheries in the Tehuantepec Gulf, Mexico. Age was estimated by counting growth rings in the scales of 266 fish. Dolphinfish size ranged from 20 to $140 \mathrm{~cm}$ Fork Length (Lf). The length-weight relationship showed negative allometry in females and positive in males. The relationship between $\mathrm{Lf}$ and scale radius for combined data of both sexes was estimated as: Lf $=37.829 \mathrm{R}$ $+3.8209\left(r^{2}=0.8276\right)$. The estimated value of the Average Percent Error (1.3\%) was considered acceptable. An annual periodicity was identified by a growth mark; marginal Increment Index indicated that a single annulus is formed yearly in April-May, which is related to the gonadosomatic index. Scales reading analysis allowed for the determination of up to four growth rings. Similar data was obtained from length frequency analysis by kernel density estimates. Results of von Bertalanffy's growth function obtained by simple non linear regression were: $L_{\infty}=125.82 \mathrm{~cm} \mathrm{Lf}, k=0.9955$ year ${ }^{-1}, t_{o}=-$ 0.037 years for females, and $L_{\infty}=126.29 \mathrm{~cm} \mathrm{Lf}, k=1.0$ year-1, $t_{0}=-0.0385$ years for males. From the analysis of monthly length frequency distribution of 7371 dolphinfish, the values obtained were: $L_{\infty}=166.5 \mathrm{~cm}, k=1.1$ year-1 for females, and $L_{\infty}=167.7 \mathrm{~cm}, k=1.3$ year $^{-1}$ for males. Longevity is estimated to be at least 4 years. No differences in growth were found between sexes. Estimated growth parameters were within the range of previous values reported for the dolphinfish at other latitudes and showed the rapid growth of the species.
\end{abstract}

Key words: Scales, von Bertalanffy, artisanal fisheries, Mexican Pacific coast

Resumen. - El dorado Coryphaena hippurus, es una de las principales especies capturadas en la pesquería artesanal en el Golfo de Tehuantepec, México. Se estimó la edad mediante el conteo de anillos de crecimiento en escamas de 266 peces. La longitud furcal (Lf) varió entre 20 y $140 \mathrm{~cm}$. La relación Lf-peso mostró una alometría negativa en hembras y positiva en machos. La relación Lf y radio de la escama para sexos combinados fue: $L f=37,829 R+3,8209\left(r^{2}=0,8276\right)$. El valor del Error Promedio Porcentual (1,3\%) se consideró aceptable. Se identificó una periodicidad anual en las marcas de crecimiento; el incremento marginal indicó que el anillo se forma en abril-mayo y coincide con el inicio del desove. El análisis de escamas permitió identificar hasta cuatro anillos de crecimiento, valores similares fueron obtenidos por medio de los estimadores de densidad de Kernel. Los resultados de la ecuación de crecimiento de von Bertalanffy obtenidos por el método de regresión no lineal simple fueron: $L_{\infty}=125,82 \mathrm{~cm} \mathrm{Lf}, k=0,9955$ año-1, $t_{0}=-0,037$ para hembras, $y L_{\infty}=126,29 \mathrm{~cm} \mathrm{Lf}, \mathrm{k}=1,0$ año-1, $\mathrm{t}_{0}=-0,0385$ para machos. Utilizando el análisis mensual de distribuciones de frecuencia-longitud de 7371 dorados se obtuvo $L_{\infty}=166,5 \mathrm{~cm}, k=1,1$ año-1 para hembras y $L_{\infty}=167,7 \mathrm{~cm}, k=1,3$ año-1 para machos. La longevidad estimada es de al menos cuatro años. No se encontró diferencia en el modelo de crecimiento por sexos. Los parámetros de crecimiento estimados se encuentran dentro del intervalo de valores reportados en otras latitudes y muestran el rápido crecimiento de la especie.

Palabras clave: Escamas, von Bertalanffy, pesca artesanal, costa del Pacífico mexicano

\section{INTRODUCCIÓN}

El dorado Coryphaena hippurus (Linnaeus 1758), también conocido como mahi-mahi o lampuga, es un depredador epipelágico migratorio que se distribuye en aguas tropicales y subtropicales de todos los océanos. Es una especie de rápido crecimiento, puede medir más de dos metros y pesar hasta $30 \mathrm{~kg}$; alcanza la madurez sexual alrededor de los 50 cm de longitud furcal (Palko et al. 1982). Esta especie, como alimento, aporta grandes volúmenes de pesca en el Mediterráneo, Caribe, Pacífico Tropical Oriental y EE. UU. (Norton \& Crooke 1994, Massutí \& Morales-Nin 1995, 
Arocha et al. 1999, Lasso \& Zapata 1999, Mahon \& Oxenford 1999, Patterson \& Martínez 1991, Schwenke \& Buckel 2008).

En el Pacífico mexicano se han reportado altas abundancias de dorado desde el Golfo de Tehuantepec hasta el sur de la Península de Baja California y en el interior del Golfo de California (Santana-Hernández 2001). No obstante, en México esta especie se encuentra reservada por ley para la pesca recreacional dentro de las 50 millas náuticas de la costa (Diario Oficial de la Federación 1995) ${ }^{1}$. Por la alta demanda del mercado y su abundancia, el dorado es una parte importante de la captura incidental de la flota artesanal en muchas partes de México, que opera con permisos para tiburón y peces demersales y que contribuye con el 55\% de las capturas artesanales (Madrid \& Beltrán-Pimienta 2001). Las pescas del dorado ocurren por flotas atuneras con redes de cerco sobre objetos flotantes, como por la pesca palangrera tiburonera. En esta última pesquería, el dorado constituye $5,2 \%$ del total de las capturas, las aguas cercanas al Golfo de Tehuantepec presentan el mayor rendimiento (SantanaHernández 2001)

El Golfo de Tehuantepec se localiza frente a la costa sur de México, en aguas del Pacífico Tropical Oriental, es una región altamente productiva y de gran complejidad ecológica. Se han observado cambios estacionales en la producción primaria y secundaria, estos están influenciados por eventos periódicos de surgencias en la temporada de nortes (noviembre-abril) y por la descarga de nutrientes de los sistemas fluvio-lagunares durante la época de lluvias (junio-octubre). Esto propicia que el Golfo sea una región importante para el desarrollo de pesquerías comerciales de pelágicos mayores (Trasviña et al. 1999), con una particular importancia sobre el atún aleta amarilla (Thunnus albacares Bonnaterre, 1788), el pez vela (Istiophorus platypterus Shaw, 1792) y el dorado (Coryphaena hippurus).

En el Pacífico mexicano, se han realizado diversas investigaciones sobre la biología del dorado. Estas incluyen aspectos sobre pesca deportiva y estadísticas de captura (Zúñiga-Flores et al. 2008), sobre dinámica poblacional (Madrid \& Beltrán-Pimienta 2001), alimentación (AguilarPalomino et al. 1998), desarrollo larval (Sánchez-Reyes 2008), y estructura genética poblacional evaluada por secuencias de ADN mitocondrial (Díaz-Jaimes et al. 2006,
Rocha-Olivares et al. 2006). Considerando que no existe heterogeneidad genética se detectaron diferencias significativas en la frecuencia de alelos en muestras del Golfo de California (Tripp-Valdez et al. 2010). Así, la información sobre los parámetros de historia de vida en las zonas de principal abundancia del dorado debe mejorar en el conocimiento sobre las unidades de manejo de esta especie.

Aunque el dorado ha sido considerado como un recurso potencial, capaz de ser explotado por cooperativas de pescadores artesanales (Anónimo 2010)², la escasez de información biológica básica y de las capturas ha sido la principal limitación para el desarrollo de un plan adecuado de manejo de la pesquería. El objetivo del presente estudio fue estimar la edad y determinar los parámetros de crecimiento del dorado, a través de las escamas de ejemplares capturados por la pesquería artesanal en el Golfo de Tehuantepec.

\section{Materiales y MÉtodos}

De diciembre de 2004 a diciembre de 2007 se realizaron muestreos mensuales en seis sitios de desembarco de la flota artesanal en el Golfo de Tehuantepec, las zonas de pesca están localizadas 5-50 millas mar afuera. La pesquería artesanal utiliza para su operación pangas de 22 a 25 pies de longitud con motor fuera de borda y son maniobradas por uno a tres pescadores. Se usan como artes de pesca palangre de superficie, curricanes de madera artesanales, redes de deriva y redes de enmalle de superficie con luz de malla de entre 3,5 y 8 pulgadas.

A cada dorado se le registró la longitud furcal (Lf) al 0,5 cm más cercano, el peso total (P) (obtenido con una sensibilidad de 0,1 kg), y peso de las gónadas (al 0,1 g más cercano). El sexo se determinó por las características externas permanentes (Collete 1995); en los peces jóvenes por examen macroscópico de las gónadas. La madurez gonádica se asignó por la observación macroscópica de las gónadas según Beardsley (1967).

El análisis de edad y crecimiento se realizó en una muestra de 266 dorados. Se removieron de 10 a 15 escamas por encima de la línea lateral al nivel de la aleta pectoral, las cuales fueron lavadas con $\mathrm{KOH}$ al $5 \%$ y montadas entre dos portaobjetos. Las imágenes de las escamas fueron obtenidas

\footnotetext{
${ }^{1}$ Diario Oficial de la Federación. 1995. Norma oficial mexicana NOM-017-PESC-1994. Para regular las actividades de Pesca deportiva en las Aguas de J urisdicción Federal de los Estados Unidos Mexicanos. N. 15-19. México, D. F.

${ }^{2}$ Anónimo 2010. Carta Nacional Pesquera. 2010. Secretaría de agricultura, ganadería, desarrollo rural, pesca y alimentación,
} México, D.F. 


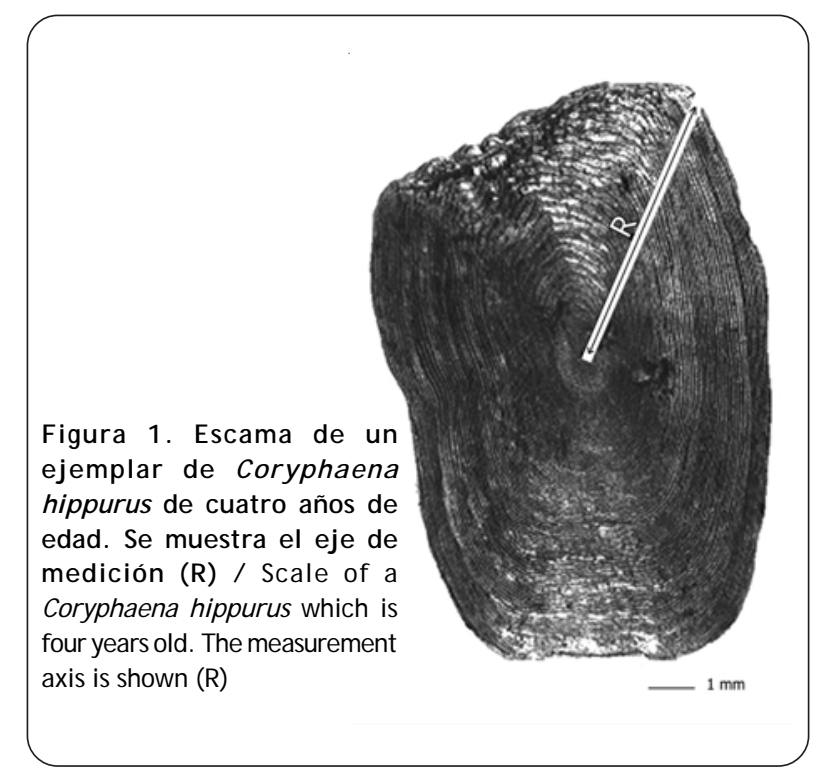

utilizando un microscopio estereoscópico Olympus ${ }^{\circledR}$ SZXTR30 acoplado a una cámara digital Leica ${ }^{\circledR}$ de 7,4 mega píxeles y a una computadora. Las imágenes digitales fueron utilizadas para medir el radio de la escama $(\mathrm{R}=$ distancia del foco al margen anterior de la escama) y la distancia del foco a cada uno de los anillos formados (Fig. 1), utilizando el programa de análisis de imagen (Motic Images Plus, version 2.0 ML, Multi Language vers. Motic Group Corporation). Las lecturas de los anillos de crecimiento se llevaron a cabo por dos lectores en diferentes tiempos siguiendo el método descrito por Holden \& Raitt (1975). El sesgo de edad y la precisión del conteo de anillos fueron examinadas usando la gráfica de error de edad (Campana et al. 1995) y el Índice del Error Promedio Porcentual (EPP) (Beamish \& Fournier 1981) entre las dos lecturas de las escamas, calculado como:

$$
E P P=\frac{1}{N} \sum_{j=1}^{N}\left[\frac{1}{R} \sum_{i=1}^{R} \frac{|X i j-X j|}{X j}\right] * 100
$$

donde $N$ es el número de muestras, $R$ es el número de veces que se leerá la muestra, $X i j$ es la edad $i$ determinada para el pez $j$ y $X j$ es el promedio de las edades determinadas para el pez $j$.

La relación entre la longitud furcal (Lf) y el radio de la escama (R) se estimó mediante el modelo lineal $(\mathrm{Lf}=\mathrm{a}+$ bR) y se comparó por sexo utilizando el análisis de covarianza (ANCOVA). Para establecer la fecha en que se formó el anillo de crecimiento y para validar su periodicidad, se estimó el incremento marginal (IM) usando la relación propuesta por Lai \& Liu (1979). La ecuación es: IM = (R - $\left.r_{n}\right) /\left(r_{n}-r_{n-1}\right)$, donde $R$ es el radio de la escama; $r_{n}$ es la distancia del foco al borde inferior del último anillo; $r_{n-1}$ es la distancia que va del foco al penúltimo anillo. Para medir el IM únicamente se utilizaron peces que presentaron al menos un anillo en sus escamas. La formación de las marcas de crecimiento en las escamas se relacionó con el índice gonadosomático [IGS= (peso de la gónada/peso del cuerpo) x 100], madurez gonádica y actividad de desove.

Para comparar y validar la lectura de escamas se utilizó el método indirecto de análisis de frecuencia de tallas a través de los estimadores de densidad por kernel (EDKs) en conjunto con el método de Bhattacharya (1967) para determinar los componentes de las curvas polimodales (Salgado-Ugarte et al. 1994, Salgado-Ugarte 2002). Para especificar el ancho de banda adecuado, se aplicó la prueba de Silverman (1981) basada en un muestreo repetitivo con reemplazamiento (bootstrap) con las rutinas para Stata 9.0 (Stata Corporation 1999) propuestas por Salgado-Ugarte et al. (2002, 2005). Esta prueba sugiere un ancho de intervalo resultante en un número de modas estadísticamente significativo en cada mes de muestreo. Los EDKs con la banda apropiada de cada mes se emplearon para determinar las clases de longitud y su distribución temporal.

El crecimiento fue descrito usando la función de crecimiento de von Bertalanffy (FCVB):

$$
L f=L_{\infty}\left\{1-\exp \left[-k\left(t-t_{0}\right)\right]\right\}
$$

donde Lf es la longitud furcal a la edad $t$; $L_{\infty}$, es la longitud asintótica; $k$, es la tasa de crecimiento; $t_{o}$, es la edad teórica que el pez debiera haber tenido para que su longitud fuese cero.

Los parámetros de crecimiento de la FCVB se estimaron para hembras y machos a partir de la regresión no lineal simple, usando las rutinas de Stata 9.0 propuestas por Salgado-Ugarte et al. (2005). Las diferencias entre FCVB por sexos se analizaron utilizando la prueba $\mathrm{T}^{2}$ de Hotelling (Bernard 1981, Salgado-Ugarte et al. 2005).

$L_{\infty} \mathrm{y} k$ fueron validados por un método independiente basado en longitudes, utilizando ELEFAN I, procedimiento incluido en el paquete FiSAT II (Gayanilo et al. 2002). Se aplicó para toda la población y para los dorados con tallas menores a $70 \mathrm{~cm}$ Lf.

La relación entre el peso total (P) y la longitud furcal (Lf) fue calculada con la función $P=a \cdot L f^{\mathrm{b}}$ (Zar 1999). Se evaluó la isometría de la pendiente obtenida con la prueba $t$-Student. El peso para todas las edades fue obtenido con 
los datos de crecimiento en longitud y la función pesolongitud. El crecimiento en peso se obtuvo sustituyendo la Lf y $L_{\infty}$ para P y $P_{\infty}$ (peso asintótico), respectivamente, en la ecuación de von Bertalanffy.

La edad límite ó longevidad de la especie $\mathrm{A}_{0,95}$ (95\% de $L_{\infty}$ ) fue determinada matemáticamente con la ecuación de Taylor (1960): $\mathrm{A}_{0,95}=\ln (1-0,95) / k+t_{0}$. Como criterio para comparar las curvas de crecimiento obtenidas por diferentes autores, se utilizó el índice de crecimiento estándar ( $\phi$ ' = $\log k+2 \log L_{\infty}$ ) (Munro \& Pauly 1983).

\section{Resultados}

Durante el periodo de estudio se midieron 7371 dorados, de ellos 3494 fueron hembras y 3877 machos. Los intervalos de Lf fueron 25,5 a $152 \mathrm{~cm}$ para los machos y de 20,5 a $129 \mathrm{~cm}$ para las hembras, ambos con al menos dos modas. Ejemplares mayores a $100 \mathrm{~cm}$ Lf se observó una proporción de machos (67\%) mayor que las hembras (33\%) (Fig. 2). Hubo diferencias significativas en la Lf por sexos (T $=12,61, P<0,01)$.

La relación lineal Lf - radio de la escama (R) fue significativa para machos y hembras $\left(r^{2}=0,9216, P<0,01\right)$. El análisis de covarianza de Lf y $\mathrm{R}$ indicó que no hay diferencias significativas entre sexos (ANCOVA: $\mathrm{F}_{(2,216)}=$ $0,13, P=0,715)$. La relación Lf - $\mathrm{R}$ fue descrita para sexos combinados por una función lineal: $L f=37,829 R+3,8209$ $\left(r^{2}=0,827, P<0,01\right)$. El conteo de los anillos de crecimiento en las escamas, se realizó a 266 peces. Se alcanzó un buen nivel de acuerdo entre dos lectores $(90,6 \%, n=241$

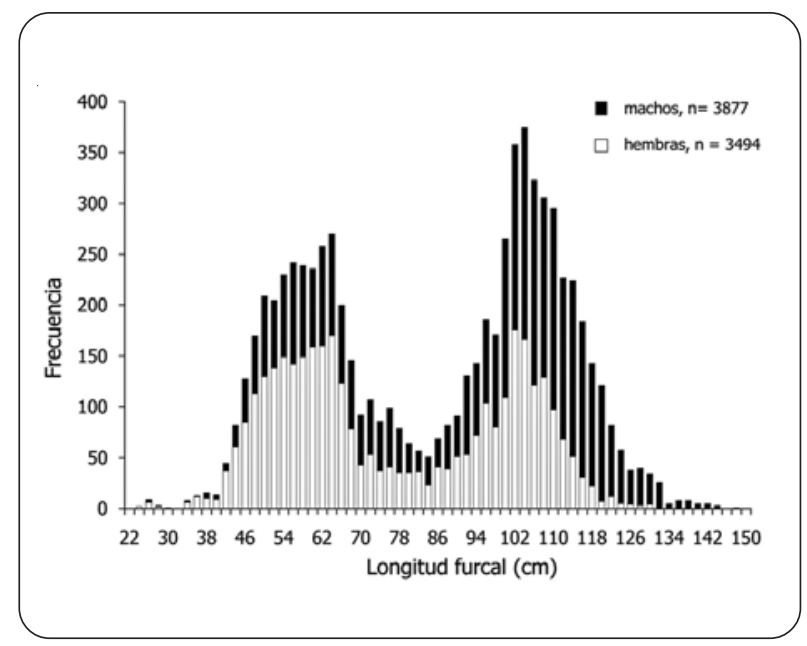

Figura 2. Distribución de frecuencia de longitud furcal para machos y hembras de Coryphaena hippurus / Furcal length frequency distribution for males and females of Coryphaena hippurus mediciones), mientras que $5,6 \%(n=15)$ presentaron dudas entre lectores y $3,76 \%(n=10)$ fueron no legibles, por lo que fueron excluidas del análisis. La comparación del número de anillos contados por los dos lectores, no indicó un sesgo apreciable; el valor del EPP (1,32\%) se consideró aceptable. Ambas lecturas pueden considerarse indistintamente; en este trabajo se usaron las lecturas estimadas por el primer lector.

La relación entre el crecimiento del pez y el crecimiento de la escama no está influenciada por el sexo (ANCOVA: $\left.\mathrm{F}_{(2,216)}=0,13, P=0,715\right)$. Así, el IM se estimó únicamente con la relación de sexos combinados y es válido tanto para hembras como para machos. Los cambios del IM promedio mensual presentaron una tendencia decreciente a partir de abril, con una abrupta disminución en mayo (Fig. 3), lo que sugiere la formación de un anillo en el borde de la escama. Posteriormente se inició la formación de una nueva zona de crecimiento, reflejándose en un aumento del IM en agosto. Lo anterior sugiere la formación de un anillo anual. El IGS mostró diferencias significativas a lo largo del año $\left(\mathrm{H}_{(11,732)}\right.$ $=610,66, P<0,001)$, con los valores más altos de septiembre a noviembre, $\mathrm{y}$ de febrero a marzo; valores significativamente bajos se observaron en mayo (Fig. 4), donde además se presentó la mayor proporción de hembras maduras. Por lo que se desprende que el periodo de formación del anillo coincide con el inicio de la temporada de desove.

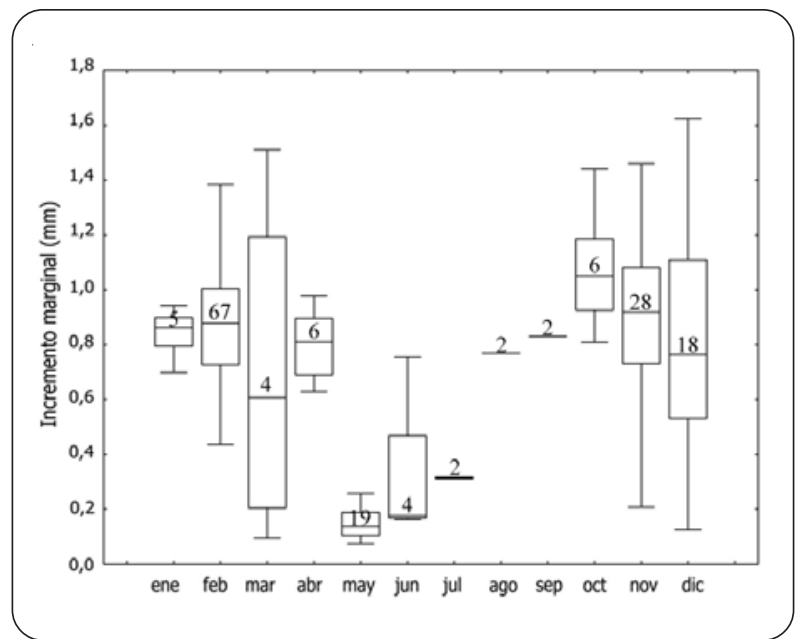

Figura 3. Variabilidad mensual del incremento marginal de anillos en escamas para sexos combinados de Coryphaena hi ppurus representado por la mediana (línea horizontal media), porcentaje (caja $=25 \%-75 \%$ ) e intervalo de los datos (líneas verticales). El número dentro de la caja indica el tamaño muestral / Monthly variability in the marginal increments of scale rings for the combined sexes of Coryphaena hippurus, represented by the median (horizontal line), percentage (box $=25 \% 75 \%$ ) and interval of data (vertical lines). Numbers inside the boxes are sample sizes 


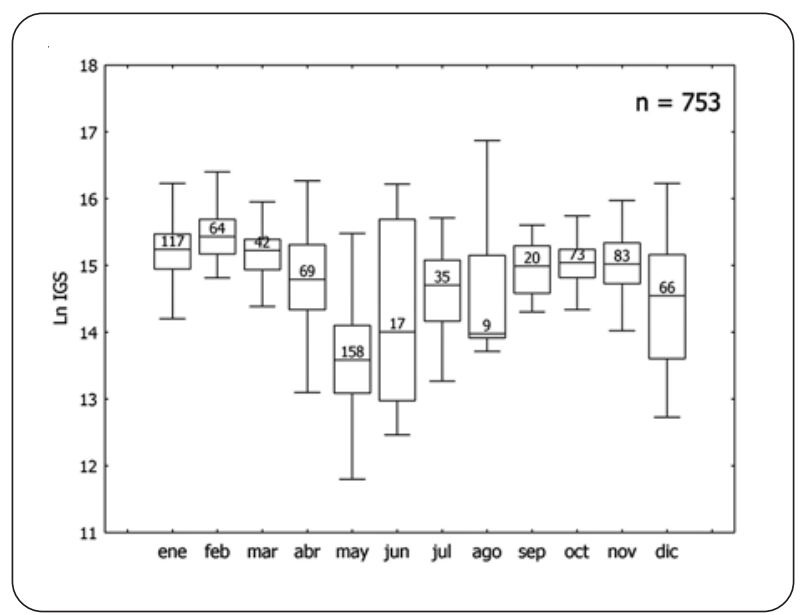

Figura 4. Variación mensual del índice gonadosomático (en logaritmo natural, Ln IGS) para hembras de Coryphaena hippurus representado por la mediana (línea horizontal media), porcentaje (caja $=\mathbf{2 5} \% \mathbf{7 5} \%$ ) e intervalo de los datos (líneas verticales). EI número dentro de la caja indica el tamaño muestral / Monthly variation of gonadosomatic index (in natural logarithm, Ln IGS) for Coryphaena hippurus females, represented by the median (horizontal line), percentage (box $=25 \% 75 \%$ ) and interval of data (vertical lines). Numbers inside the boxes are sample sizes

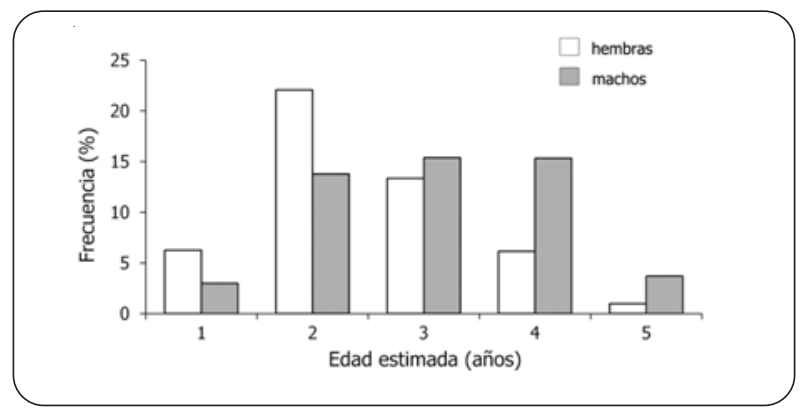

Figura 5. Distribución de frecuencia de individuos (machos y hembras) por edad estimada (años) de Coryphaena hippurus en las capturas artesanales / Frequency distribution of individuals (males and females) per estimated age (years) of Coryphaena hippurus in artisanal catches

Durante la lectura de las estructuras duras, se registró que $40 \%$ de las escamas no presentaron anillo de crecimiento y se consideraron en edad $0+$. El análisis de escamas identificó cuatro grupos de edad para ambos sexos (Tabla 1). Los EDKs para el mes de noviembre con banda bootstrap de Silverman fueron polimodales y los valores medios obtenidos por Bhattacharya para cada componente fueron: 53,$27 ; 110,02 ; 126,41 ; 137,33$ para hembras y 53,0; 107,24; 122,$81 ; 133,08$ para machos. Estas medias muestran pocas diferencias con los valores obtenidos por la lectura de escamas. Más de la mitad de la captura artesanal correspondió a las edades de $0+$ y 1 año, con tallas promedio de $58 \mathrm{~cm} \mathrm{Lf} \mathrm{(Fig.} \mathrm{5).} \mathrm{En} \mathrm{la} \mathrm{Tabla} 2$ se presentan los
Tabla 1. Edad (años) determinada mediante la lectura de anillos en escamas de Coryphaena hippurus en el Golfo de Tehuantepec; $\mathbf{n}=$ número de escamas analizadas, promedio de la longitud furcal (Lf) y su desviación estándar (DE) / Age (years) determined through reading scale rings of Coryphaena hippurus in the Gulf of Tehuantepec; $n=$ number of scales, average of furcal length (Lf) and its standard deviation (DE)

\begin{tabular}{lrrrrrrrrr}
\hline & \multicolumn{4}{c}{ Machos } & \multicolumn{4}{c}{ Hembras } \\
Edad & $\mathrm{n}$ & $\begin{array}{c}\text { Promedio } \\
\text { (Lf) }\end{array}$ & $\begin{array}{r}\text { DE } \\
\text { Intervalo } \\
\text { Lf }(\mathrm{cm})\end{array}$ & $\begin{array}{c}\mathrm{n} \\
\text { (Lf) }\end{array}$ & $\begin{array}{c}\text { Promedio } \\
\text { DE }\end{array}$ & $\begin{array}{c}\text { Intervalo } \\
\text { Lf }(\mathrm{cm})\end{array}$ \\
\hline $0+$ & 32 & 43,0 & 6,71 & $37-54$ & 79 & 46,9 & 10,58 & $26-59$ \\
1 & 17 & 57,7 & 12,19 & $57-84$ & 18 & 58,4 & 9,46 & $46-76$ \\
2 & 7 & 103,9 & 10,49 & $89-114$ & 11 & 103,7 & 7,32 & $91-114$ \\
3 & 29 & 111,8 & 6,94 & $96-124$ & 19 & 111,4 & 3,97 & $104-120$ \\
4 & 27 & 125,9 & 4,91 & $120-135$ & 17 & 125,5 & 3,97 & $120-135$ \\
\hline
\end{tabular}

Tabla 2. Valores de los parámetros de la función de crecimiento de von Bertalanffy, obtenidos por diferentes métodos. LFA: análisis frecuencia de longitud (ELEFAN I); $\mathbf{S}=$ lectura en escamas; K: análisis de frecuencia de longitud por EDKs (regresión no lineal); $\mathbf{n}=$ número de organismos; $\mathbf{L}_{\infty}=$ longitud asintótica ( $L f$, $\mathrm{cm}) ; \mathbf{k}=$ coeficiente de crecimiento en un año; $t_{0}=$ longitud a la edad $0 ; r^{2}$ aj = coeficiente de regresión ajustado; * según Beverton y Holt (1957); $\mathbf{R n}=$ índice de bondad de ajuste; $\phi^{\prime}=$ índice de crecimiento estándar / Von Bertalanffy growth parameters for Coryphaena hippurus were calculated by different methods. LFA: length-frequency analysis (ELEFAN I); $\mathrm{S}=$ scale readings; $\mathrm{K}$ : length frequency analysis by EDKs (non linear regression); $\mathrm{n}=$ number specimens; $L_{\infty}=$ asymptotic length ( $\left.\mathrm{cm} \mathrm{Lf}\right), \mathrm{k}=$ growth coefficient in one year, $t_{0}=$ length at age $=0, r^{2}$ aj $=$ adjusted regression coefficient, $*$ from Beverton and Holt (1957), Rn $=$ goodness-of-fit index; $\phi^{\prime}=$ growth performance index

\begin{tabular}{lcccccccc}
\hline Población & Método & $n$ & $L_{\infty}$ & $k$ & $t_{o}$ & $\mathrm{r}^{2}$ aj & $\mathrm{Rn}$ & $\phi^{\prime}$ \\
\hline Hembras & $\mathrm{S}$ & 136 & 125,83 & 0,9955 & $-0,037$ & 0,99 & & 4,20 \\
Machos & $\mathrm{S}$ & 105 & 126,28 & 1,0012 & $-0,385$ & 0,99 & & 4,20 \\
Todos & $\mathrm{S}$ & 241 & 126,03 & 0,9506 & $-0,0319$ & 0,99 & 4,18 \\
Hembras & $\mathrm{K}$ & 253 & 135,51 & 1,0311 & 0,0568 & 0,99 & & 4,28 \\
Machos & $\mathrm{K}$ & 415 & 139,98 & 1,0233 & 0,0529 & 0,99 & & 4,30 \\
Hembras & LFA & 534 & 166,5 & 1,1 & $-0,052^{*}$ & - & 0,152 & 4,48 \\
Machos & LFA & 734 & 166,7 & 1,3 & $-0,052^{*}$ & - & 0,132 & 4,56 \\
Jóvenes & LFA & 698 & 67,2 & 1,84 & $-0,074^{*}$ & - & 0,150 & 3,92 \\
\hline
\end{tabular}

parámetros de crecimiento estimados utilizando la regresión no lineal. No se encontraron diferencias en el modelo de crecimiento por sexos $\left(\mathrm{T}^{2}=0,803, P>0,01\right)$ (Fig. 6). La longevidad se determinó en 4 años de edad.

Los resultados obtenidos de versiones discretizadas de los EDKs mensuales para las capturas de 2005 a 2007 fueron introducidos a la rutina ELEFAN I del FiSAT. Por este método se ajustó una curva de crecimiento con tasa constante de crecimiento sin oscilaciones estacionales 


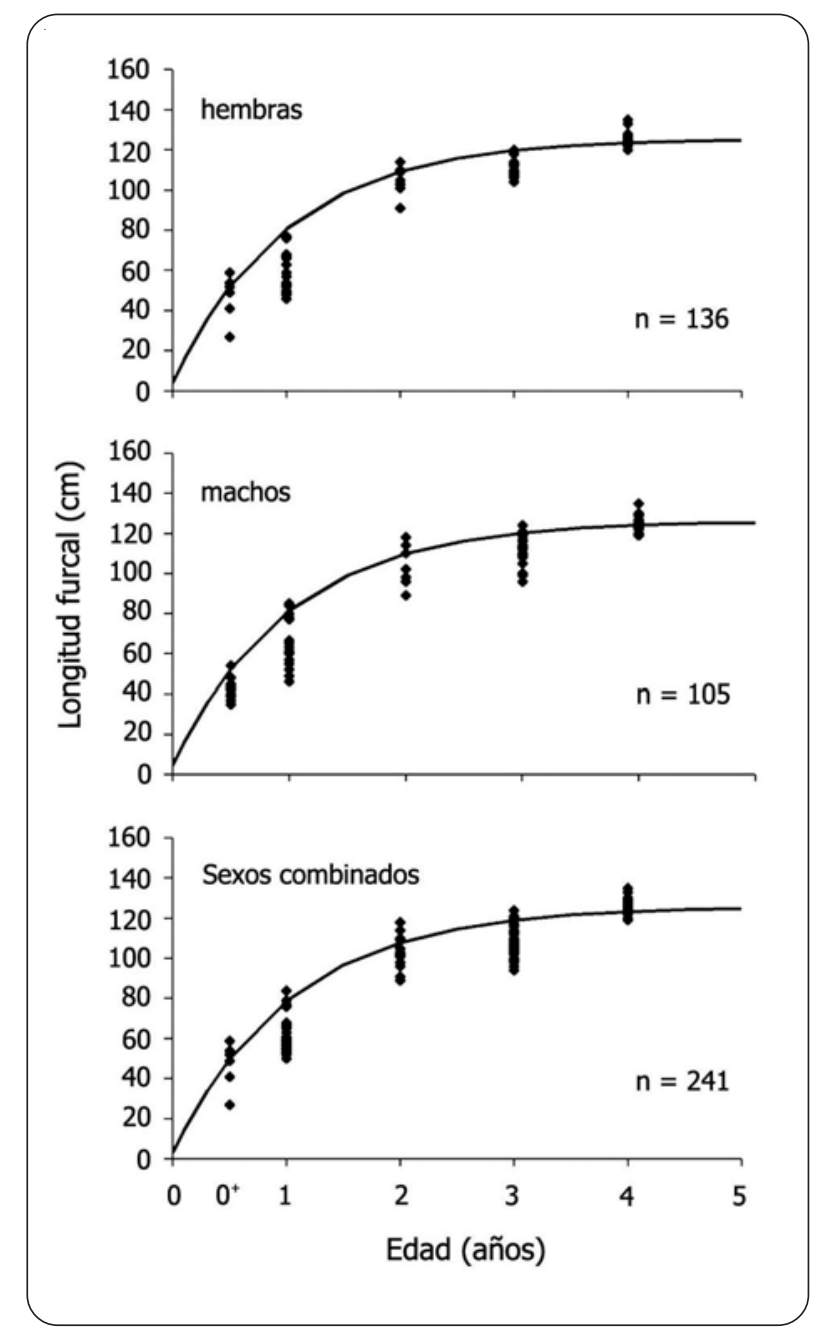

Figura 6. Curvas de crecimiento en longitud (función de von Bertalanffy) para hembras, machos y sexos combinados de Coryphaena hippurus determinadas por los anillos de crecimiento en escamas / Growth curves (von Bertalanffy function) for male, female and combined samples of Coryphaena hippurus determined by the scale's growth rings

que pasa por la mayoría de los picos modales. La aplicación de este procedimiento a dorados jóvenes (20 a $70 \mathrm{~cm} \mathrm{Lf)} \mathrm{proporcionó} \mathrm{un} \mathrm{parámetro} \mathrm{de} \mathrm{curvatura}$ relativamente elevado $\left(k=1,84\right.$ año $\left.^{-1}\right)$ (Tabla 2).

La relación entre Lf y el peso total (Pt) fue alométrica, positiva en los machos: $\mathrm{Pt}=4 \times 10^{-6} L f^{3,1435}(t=3,486, P<$ $0,01)$ y negativa en hembras: $\mathrm{Pt}=1,2 \times 10^{-5} L f^{2,8482}(t=$ $3,469, P<0,01)$. Las curvas ajustadas sugieren que los machos fueron más pesados que las hembras a partir de los $35 \mathrm{~cm}$ Lf (Fig. 7) $(\mathrm{T}=8,57, P<0,01)$. La curva de crecimiento de los valores estimados del peso asintótico fueron: $P_{\infty}=38,83 \mathrm{~kg}$ para machos y $P_{\infty}=25,26 \mathrm{~kg}$ en las hembras.

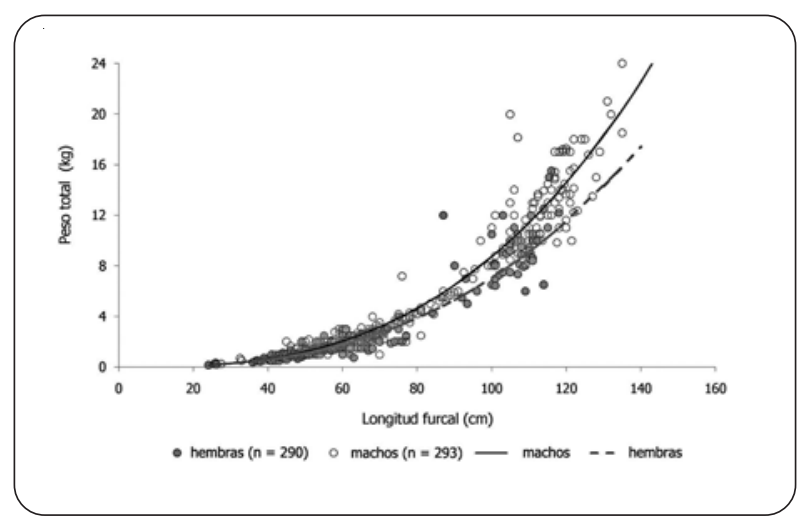

Figura 7. Relación entre el peso y la longitud furcal para machos y hembras de Coryphaena hippurus / The relationship between body weight and furcal length for males and females of Coryphaena hippurus

\section{Discusión}

El examen de las escamas permitió observar y discriminar con claridad los anillos de crecimiento, lo que se corrobora con los resultados entregados para esta especie por diferentes autores (Beardsley 1967, Massutí et al. 1999, Schwenke \& Buckel 2008). Los trabajos anteriores mencionan que el nuevo anillo se forma por la disminución de la temperatura durante el invierno. Al respecto Hassler \& Hogarth (1977) señalan que Coryphaena hippurus es muy sensible a los cambios de temperatura los cuales afectan su tasa de alimentación, misma que disminuye por debajo de los $23^{\circ} \mathrm{C}$ y cesa a $18^{\circ} \mathrm{C}$. En zonas tropicales es de esperar un crecimiento continuo debido a una tasa de crecimiento alta y la disponibilidad de alimento durante todo el año (Uchiyama et al. 1986). Los resultados obtenidos en el presente trabajo señalan la formación del anillo de crecimiento a partir abril y hasta mayo. Este evento puede atribuirse a las variaciones del metabolismo causadas por la madurez gonádica y los gastos de energía producto de la actividad de desove. Alejo-Plata et al. (2011) mencionan que en el Golfo de Tehuantepec, C. hippurus presenta la talla de primera madurez $\left(\mathrm{Lf}_{50 \%}\right)$ a $\operatorname{los} 48,38 \pm 0,84 \mathrm{~cm}$ Lf, y sigue una estrategia reproductiva asincrónica, y con el primer desove masivo en mayo. Esto confirma su rápido crecimiento en las primeras etapas de vida, antes de la madurez sexual y apoya la hipótesis que el lento crecimiento somático es debido a que gran parte de la energía destinada a este proceso fisiológico es utilizada en la reproducción. Las lluvias y el aumento en la disponibilidad de alimentos por la descarga de sistemas fluvio-lagunares ocurren a partir de junio. Por tanto estos son responsables del incremento somático posterior a la reproducción y por tanto después de que se formo el anillo. 
Tabla 3. Parámetros de crecimiento de von Bertalanffy calculados para Coryphaena hippurus en estudios previos en diferentes regiones geográficas. $L_{\infty}=$ longitud asintótica $(L f, \mathbf{c m}) ; k=$ coeficiente de crecimiento en un año; $\mathbf{t}_{\mathrm{o}}=$ longitud a la edad =0; $\phi^{\prime}$ = índice de crecimiento estándar / Von Bertalanffy growth parameters calculated for Coryphaena hippurus from previous studies at different geographical regions. $\mathrm{L}_{\infty}=$ asymptotic length $(\mathrm{cm} \mathrm{Lf})$; $\mathrm{k}=$ growth coefficient in one year; $\mathrm{t}_{\mathrm{o}}=$ length at age $=0 ; \phi^{\prime}=$ growth performance index

\begin{tabular}{llccclll}
\hline Área & Grupo & $L_{\infty}$ & $k$ & $t_{o}$ & Método & $\phi$ & Referencia \\
\hline Panamá y Colombia & Todos & 194,0 & 0,91 & 0,105 & Estructura tallas & 4,50 & Lasso \& Zapata, (1999) \\
Carolina del Norte & Todos & 159,7 & 0,40 & $-0,964$ & escamas & 4,00 & Rose \& Hassler (1968) \\
& & 123,7 & 1,1 & $-0,116$ & Otolitos, escamas & 4,22 & Schwenke \& Buckel (2008) \\
Carolina del Norte & Hembras & $1,9,9$ & 1,18 & $-0,089$ & & 4,30 & \\
& Machos & 129,9 & & 4,20 & \\
& Todos & 129,9 & 1,08 & $-0,086$ & & 4,27 & Beardsley (1967) \\
Estrecho de Florida & Todos & 164,0 & 0,68 & 0,155 & Escamas & 4,47 & Massutí et al. (1999) \\
Mediterráneo & Todos & 102,4 & 1,9 & 0,023 & Otolitos & 4,60 & Bentivoglio (1988) \\
Golfo de México & Todos & 194,0 & 1,12 & 0,033 & Otolitos & 4,70 & Rivera \& Appeldoorn (2000) \\
Puerto Rico & Todos & 145,7 & 2,19 & $-0,46$ & Otolitos, escamas & 4,70 & Oxenford (1999) \\
Barbados & Hembras & 122,1 & 3,43 & 0,063 & Otolitos & 4,90 & \\
& Machos & 126,0 & 5,24 & 0,089 & Otolitos & 4,90 & \\
& Todos & 120,8 & 3,49 & 0,055 & Otolitos & 4,70 & \\
Ecuador & Todos & 195,0 & 0,41 & & Estructura tallas & 4,19 & Patterson \& Martínez (1991) \\
\hline
\end{tabular}

La correspondencia de las medias de longitud a cada edad obtenida, a partir de la lectura de escamas con las medias de los componentes gaussianos obtenidos de los EDKs multimodales, permite validar el método directo de estimación de edades. Los resultados obtenidos resaltan la confiabilidad y dan evidencia de las ventajas del uso de los EDKs en combinación con métodos originalmente diseñados para histogramas (ELEFAN I).

Los parámetros de crecimiento para esta especie sugieren una tasa de crecimiento rápido durante el primer año de vida tanto en longitud ( $\sim 58 \mathrm{~cm} \mathrm{Lf}$ ) como en peso ( $\sim 3 \mathrm{~kg})$. La característica más notable acerca de su crecimiento es la amplia gama de tallas de los organismos con un anillo de crecimiento. Los dorados que fueron capturados durante mayo-junio, son más pequeños que los capturados en noviembre-febrero. De acuerdo a la progresión modal, la afluencia de peces pequeños a la zona de pesca en mayo, puede representar la entrada de una nueva cohorte. El muestreo de dos cohortes sucesivas pertenecientes a una misma clase de edad es probable, dada la naturaleza altamente migratoria del dorado, esto se ha reportado para las poblaciones del Atlántico (Massutí \& Morales-Nin 1995) y el Pacífico norte (Kraul 1999).

En la literatura se ha referido una edad de 3 años para los dorados capturados en la costa de Carolina del Norte (Beardsley 1967, Schwenke \& Buckel 2008) y Mar Mediterráneo (Massutí et al. 1999), de 4 años para Florida
EUA (Rose \& Hassler 1968) y de 5 años para las costas de Japón (Kojima 1965). En este estudio se determinó una edad de 4 años, sin embargo es probable la existencia de hasta 5 marcas en las escamas, debido a que se registraron cinco dorados machos $(\mathrm{Lf}>140 \mathrm{~cm}$ ) con un borde después del cuarto anillo. Lo anterior sugiere que en el Golfo de Tehuantepec los dorados pueden alcanzar los 5 años de edad, no obstante estos organismos grandes están siendo poco accesibles a las artes de pesca de la flota ribereña. La distribución de tallas por mes mostró la incorporación permanente de individuos pequeños a la zona de pesca que corresponde al crecimiento de la población a lo largo del año.

Para la determinación de los parámetros de crecimiento se consideraron únicamente organismos con al menos un anillo bien formado. El valor de $L_{\infty}$ se observó subestimado debido al escaso número de escamas de ejemplares mayores a $120 \mathrm{~cm}$ Lf, no obstante representa la longitud máxima promedio de las capturas artesanales, y $k$ estaría representando la tasa de crecimiento de dorados adultos. Estos parámetros se encuentran en el intervalo de valores reportado para esta especie en diferentes regiones geográficas (Tabla 3).

La progresión modal en las capturas de dorados juveniles mostró una rápida tasa de crecimiento, que se vió reflejada en el aumento de casi el doble de longitud media mensual desde finales de abril hasta principios de julio. Este rápido crecimiento ha sido reportado en otras latitudes (Murray 
1985, Oxenford \& Hunte 1986, Massutí et al. 1999) y en cultivos de dorado (e.g., Bennetti et al. 1995). Esta especie es de vida corta, con valores altos en la tasa de crecimiento $\left(k=1,3\right.$ año $\left.^{-1}\right)$, que alcanza la madurez sexual antes de cumplir un año de edad. Las distribuciones multimodales obtenidas en los EDKs indican cohortes que pudieron ser seguidas por el método ELEFAN I y que llevan a estimaciones de crecimiento válidas para el intervalo de tallas comprendido en las muestras.

La relación peso-longitud mostró una alometría negativa en hembras y positiva en los machos. La diferencia en el peso para una longitud dada, parece aumentar gradualmente con el incremento de la longitud, esto va de acuerdo con el dimorfismo sexual de $C$. hippurus. En esta especie es característica la presencia de una pronunciada cresta ósea en los machos que comienza a formarse en individuos de alrededor de $40 \mathrm{~cm}$ Lf (Beardsley 1967). La alometría en esta especie ha sido observada por Palko et al. (1982), Massutí et al. (1999), Oxenford (1999). Los parámetros obtenidos para machos $\left(P_{\infty}=38,83 \mathrm{~kg}\right)$ y hembras $\left(P_{\infty}=25,26 \mathrm{~kg}\right)$ muestran un crecimiento en peso significativamente diferente entre los sexos, lo cual comienza a evidenciarse a partir de la edad 1 a favor de de los machos.

Las diferencias en los parámetros de crecimiento para esta especie en distintas áreas geográficas se deben, entre otras razones, a los métodos de laboratorio, a diferencias genéticas, a condiciones ambientales (e.g., temperatura del agua, disponibilidad de alimento, niveles de explotación) (Davis et al. 2008) y a la escasez de organismos pequeños y grandes en las capturas (Campana 2001). Estas diferencias se compensan con la evaluación del índice de crecimiento estándar ( $\phi$ '), que muestra valores similares entre las poblaciones, con un coeficiente de variación del $6 \%$, lo que indica un mismo patrón de crecimiento (Tabla 3). Utilizando únicamente otolitos sagita los valores son más altos porque las edades y crecimiento fueron determinados usando incrementos diarios en todas las tallas del pez, este método puede subestimar la edad de los peces grandes (Massutí et al. 1999). En consecuencia, el uso de escamas parece ser desde el punto de vista de la preparación de las escamas y resultados obtenidos, el mejor método para la determinación de edad en peces adultos. Varios autores (Massutí et al. 1999, Rivera \& Appeldoorn 2000, Schwenke \& Buckel 2008) mencionan que al usar de manera combinada incrementos diarios en otolitos de peces juveniles y anillos en escamas de dorados adultos, los parámetros $k$ y $L_{\infty}$ proporcionan resultados más cercanos al crecimiento del dorado.
Otros estudios han determinado que la estructura del stock y los patrones de migración del dorado son complejos (Oxenford \& Hunte 1986, Rivera \& Appeldoorn 2000, Díaz-Jaimes et al. 2006, Tripp-Valdez et al. 2010). Así, para una especie que se captura tanto por la pesca deportiva como por la pesca comercial, resulta esencial obtener información sobre los parámetros de historia de vida a lo largo de las zonas de captura, lo que constituye información útil para los planes de manejo de la pesquería.

\section{Agradecimientos}

A los pescadores de Oaxaca y Chiapas. A los colegas del laboratorio de ictiología y biología pesquera de la Universidad del Mar por su apoyo en el trabajo de campo. A Pedro Cervantes por la revisión del documento y a los árbitros anónimos. El trabajo fue financiado por el Fondo Sectorial SAGARPA-CONACYT proyecto 225-2003 y el Gobierno del Estado de Oaxaca-CONAPESCA (2IR0502). A PROMEP. Este estudio ha sido parte de la Tesis Doctoral del primer autor en el Posgrado en Ciencias Biológicas, Universidad Nacional Autónoma de México.

\section{LiTERATURA CITADA}

Aguilar-Palomino B, F Galván-Magaña, A AbitiaCardenas, A Muhlia-Melo \& J Rodríguez-Romero. 1998. Aspectos alimentarios del dorado Coryphaena hippurus Linnaeus, 1758 en cabo San Lucas, Baja California Sur, México. Ciencias Marinas 24: 253-265.

Alejo-Plata C, P Díaz-Jaimes \& IH Salgado-Ugarte. 2011. Sex ratios, size at sexual maturity, and spawning seasonality of dolphinfish (Coryphaena hippurus) captured off the Gulf of Tehuantepec, Mexico. Fisheries Research 110: 207216.

Arocha F, L Marcano, A Lárez, D Altuve \& J Alio. 1999. The fishery, demographic size structure and oocyte development dolphinfish, Coryphaena hippurus, in Venezuela and adjacent waters. Scientia Marina 63(3-4): 401-409.

Bhattacharya CG. 1967. A simple method of resolution of a distribution into Gaussian components. Biometrics 23: 115135.

Beamish RJ \& DA Fournier. 1981. A method for comparing the precision of a set of age determinations. Canadian Journal of Fisheries and Aquatic Sciences 3: 982-983.

Beardsley GL Jr. 1967. Age, growth and reproduction of the dolphin, Coryphaena hippurus, in the Straits of Florida. Copeia 1967: 441-451.

Benetti DD, ES Iversen \& AC Ostrowski. 1995. Growth rates of captive dolphin, Coryphaena hippurus, in Hawaii. Fishery Bulletin 93(1): 152-157. 
Bentivoglio AA. 1988. Investigations into the growth, maturity, mortality rates and occurrence of the dolphin (Coryphaena hippurus, Linnaeus) in the Gulf of Mexico. M. Sc. Thesis, University College of North Wales, Bangor, 37 pp.

Bernard DR. 1981. Multivariate analysis as a means of comparing growth in fish. Canadian Journal of Fisheries and Aquatic Sciences 38: 233-236.

Campana SE. 2001. Accuracy, precision and quality control in age determination, including review of the use and abuse of age validation methods. Journal of Fish Biology 59: 197242.

Campana SE, MC Annand \& JI McMillan. 1995. Graphical and statistical methods for determining the consistency of age determinations. Transactions of the American Fisheries Society 124: 131-138.

Collete BB. 1995. Coryphaenidae. En: Fisher W, F Krupp, W Schneider, C Sommer, KE Carpenter \& VH Niem (eds). Guía FAO para la identificación de especies para los fines de la Pesca. Pacífico Centro-Oriental, volumen II. Vertebrados - Parte 1, pp. 1036-1038. FAO, Roma.

Davis AC, D Brophy, P Megalofonov \& E Gosling. 2008. Age estimation in calcified calcareous structures; preliminary finding of an inter laboratory comparison. Collective Volume of Scientifics Papers ICCAT 62(3): 899910.

Díaz-Jaimes P, M Uribe-Alcocer, S Ortega-García \& JD Durand. 2006. Spatial and temporal mitochondrial DNA genetic homogeneity of dolphinfish populations (Coryphaena hippurus) in the eastern central Pacific. Fisheries Research 80: 333-338.

Gayanilo FC, P Sparre \& D Pauly. 2002. FAO-ICLARM Stock Assessment Tools (FiSAT). Software versión 1.2.0. FAO. Roma.

Hassler WW \& WT Hogarth. 1977. The growth and culture of dolphin, Coryphaena hippurus, in North Carolina. Aquaculture 12: 115-122.

Holden MJ \& DFS Raitt. 1975. Manual de Ciencia Pesquera. Parte 2.- Métodos para investigar los recursos y su aplicación. ONU/FAO Documento Técnico de Pesca 115, Rev. 1: 1-255.

Kojima S. 1965. Studies on the fishing conditions of the dolphin Coryphaena hippurus L., in the western regions of the Sea of Japan-X. Results on tagging experiment. Bulletin of the Japanese Society of Fisheries Oceanography 31: 575-578.

Kraul S. 1999. Seasonal abundance of the dolphinfish, Coryphaena hippurus, in Hawaii and the tropical Pacific Ocean. Scientia Marina 63(3-4): 261-266.

Lai HL \& HC Liu. 1979. Age and growth of Lutjanus sanguineus in the Arafura Sea and North West Shelf. Acta Oceanographica Taiwanica 10: 160-171.
Lasso J \& L Zapata. 1999. Fisheries and biology of Coryphaena hippurus (Pisces: Coryphaenidae) in the Pacific coast of Colombia and Panama. Scientia Marina 63(3-4): 387-399.

Madrid JV \& R Beltrán-Pimienta. 2001. Length, weight and sex of the dolphin fish Coryphaena hippurus (Perciformes: Coryphaenidae), of the littoral of Sinaloa, Nayarit and Baja California Sur, Mexico. Revista de Biología Tropical 49: 931-938.

Mahon R \& HA Oxenford. 1999. Precautionary assessment and management of dolphinfish in the Caribbean. Scientia Marina 63(3-4): 429-438.

Massutí E \& B Morales-Nin. 1995. Seasonality and reproduction of dolphinfish (Coryphaena hippurus) in the western Mediterranean. Scientia Marina 59: 357-364.

Massutí E, B Morales-Nin \& J Moranta. 1999. Otolith microstructure, age, and growth patterns of dolphin, Coryphaena hippurus, in the western Mediterranean. Fishery Bulletin 97: 891-899.

Munro JL \& D Pauly. 1983. A simple method for comparing the growth of fishes and invertebrates. Fishbyte 1: 5-6.

Murray PA. 1985. Growth and mortality in the dolphinfish Coryphaena hippurus caught off Saint Lucia, W.I. FAO Fishery Report 327: 47-153.

Norton JG \& SJ Crooke. 1994. Occasional availability of dolphin, Coryphaena hippurus, to southern California Commercial Passenger Fishing Vessel Anglers: Observations and Hypotheses. California Cooperation Oceanic Fishery Investigation 35: 230-239.

Oxenford H. 1999. Biology of the dolphinfish (Coryphaena hippurus) in the western central Atlantic: a review. Scientia Marina 63(3-4): 277-301.

Oxenford HA \& W Hunte. 1986. A preliminary investigation of the stock structure of the dolphin, Coryphaena hippurus, in the western central Atlantic. Fishery Bulletin 84: 451460 .

Palko B, G Beardsley \& W Richards. 1982. Synopsis of biological data on dolphin-fishes Coryphaena hippurus and Coryphaena equiselis L. NOAA Technical Report NMFS 443: 1-28.

Patterson KR \& J Martínez. 1991. Explotation of the dolphin-fish Coryphaena hippurus L. off Ecuador: analysis by length-based virtual population analysis. Fishbyte 9: 21-23.

Rivera GA \& RS Appeldoorn. 2000. Age and growth of dolphinfish, Coryphaena hippurus, off Puerto Rico. Fishery Bulletin 2: 345-352.

Rocha-Olivares A, M Bobadilla-Jiménez, S Ortega-García, N Saavedra-Sotelo \& JR Sandoval-Castillo. 2006. Variabilidad mitocondrial del dorado Coryphaena hippurus en poblaciones del Pacífico. Ciencias Marinas 32: 569-578. 
Rose CD \& WW Hassler. 1968. Age and growth of the dolphin, Coryphaena hippurus (Linnaeus), in North Carolina waters. Transactions of the American Fisheries Society 97: 271276.

Salgado-Ugarte IH. 2002. Suavización no paramétrica para el análisis de datos, 139 pp. Facultad de Estudios Profesionales Zaragoza y DGAPA, UNAM, México.

Salgado-Ugarte IH, M Shimizu \& T Taniuchi. 1994. Semigraphical determination of Gaussian components in mixed distributions. Stata Technical Bulletin 18: 15-27.

Salgado-Ugarte IH, JL Gómez-Márquez \& B PeñaMendoza. 2005. Métodos actualizados para Análisis de Datos Biológico-Pesqueros, 240 pp. Facultad de Estudios Superiores Zaragoza y DGAPA, UNAM, México.

Salgado-Ugarte IH, M Shimizu, T Taniuchi \& K Matsushita. 2002. Nonparametric assessment of multimodality for size frequency distributions. Asian Fisheries Science 15: 295-303.

Sánchez-Reyes N. 2008. Distribución de larvas de dorado Coryphaena hippurus (Linnaeus, 1758) y Coryphaena equiselis (Linnaeus, 1758) en el Pacífico Oriental mexicano. Tesis Maestría en Ciencias, Centro Interdisciplinario de Ciencias Marinas-Instituto Politécnico Nacional, La Paz, 155 pp.

Santana-Hernández H. 2001. Estructura de la comunidad de pelágicos mayores capturados con palangre en el Pacífico mexicano (1983-1996) y su relación con la temperatura superficial del mar. Tesis Doctoral, Facultad de Ciencias, Universidad de Colima, Colima, 122 pp.
Schwenke KL \& JA Buckel. 2008. Age, growth, and reproduction of dolphinfish (Coryphaena hippurus) caught off the coast of North Carolina. Fishery Bulletin 106: 8292.

Silverman BW. 1981. Using kernel density estimates to investigate multimodality. Journal of the royal Statistical Society B 43: 97-99.

Taylor CC. 1960. Temperature, growth and mortality -The Pacific cockle. Journal du Conseil 26(1): 177-224.

Trasviña A, D Lluch-Cota, AE Filonov \& A Gallegos. 1999. Oceanografía y El Niño. Capitulo 3. En: V Magaña (ed). Los impactos de El Niño en México, pp. 69-102. UNAM, México.

Tripp-Valdez MA, FJ García de León, S Ortega-García, D Lluch-Cota, J López-Martínez \& P Cruz. 2010. Population genetic structure of dolphinfish (Coryphaena hippurus) in the Gulf of California, using microsatellite loci. Fisheries Research 105: 172-177.

Uchiyama JH, RK Burch \& SA Kraul. 1986. Growth of dolphins, Coryphaena hippurus and C. equiselis, in Hawaiian waters as determined by daily increments in otoliths. Fishery Bulletin 84: 186-191.

Zar JH. 1999. Biostatistical analysis, 718 pp. Prentice Hall, Upper Saddle River.

Zúñiga-Flores MS, S Ortega-García \& A Klett-Traulsen. 2008. Interannual and seasonal variation of dolphinfish (Coryphaena hippurus) catch rates in the southern Gulf of California, Mexico. Fisheries Research 94: 13-17.

Recibido el 20 de diciembre de 2010 y aceptado el 28 de febrero de 2011 